\title{
Editorial
}

\section{Salud mental adolescente. Una invitación a pensar en su salud mental desde miradas positivas}

\author{
Cristóbal Ovidio Muñoz Arroyave \\ Universidad CES \\ Colombia
}

Forma de citar:

Muñóz Arroyave, C.0. (2020). Salud mental adolescente. Una invitación a pensar en su salud mental desde miradas positivas. Rev.CES Psico, 13(1), i-iii.

\section{Open access}

\section{(c) Copyright}

Licencia creative commons

Etica de publicaciones

Revisión por pares

Gestión por Open Journal System DOl: http://dx.doi.org/10.21615/

cesp.13.1.11

ISSN: 2011-3080

Sobre los autores:

1. Doctor (c) en Epidemiología y Bioestadística. Magister en Educación y Desarrollo. Psicólogo. Docente investigador Universidad CES.
Comparte
En la actualidad, muchos adolescentes mueren de forma prematura debido a accidentes, suicidios, violencia, complicaciones relacionadas con el embarazo y enfermedades prevenibles o tratables; y otras enfermedades graves, como el consumo de tabaco, las infecciones de transmisión sexual y los malos hábitos alimentarios y de ejercicio, comienzan en la adolescencia, lo que ha llevado a considerar esta población como un grupo vulnerable o en riesgo (Herrera Santi, 1999; Organización Mundial de la Salud -OMS-, s.f.; UNICEF, 2011).

No obstante, las transformaciones dadas en el campo de la salud y el posicionamiento del movimiento de desarrollo positivo en la explicación del comportamiento adolescente han cimentado nuevas preguntas que permiten transitar de los modelos biomédicos y comportamentales hacia enfoques complejos y proactivos.

La dominancia tanto del modelo biomédico que concibe la enfermedad mental como producto de factores anatómicos, fisiológicos o de entidades externas que afectan el funcionamiento orgánico, como del modelo comportamental y sus estudios sobre factores de riesgos, sean estos ambientales o individuales (Restrepo \& Jaramillo, 2012), hoy se encuentra minada por un sin número de investigaciones que reconocen al individuo como un sujeto con capacidad para agenciar sus decisiones y comprometerse con ellas.

Sumada a esta mirada sobre la salud mental aparece el movimiento conocido como desarrollo positivo, que concibe al adolescente como un sujeto activo, históricamente construido, en interacción continua con los contextos donde se desenvuelve, con capacidad de cuestionar, elegir y decidir los modos en que desea posicionarse en el mundo en una lógica de compromiso consigo mismo y el otro; teorías como el propósito de vida (Damon, 2008; Kashdan \& Mcknight, 2009), la iniciativa personal (Larson, 2000), la satisfacción vital (Frisch, 2000) y la automotivación (Hui \& Tsang, 2012) hacen parte de este movimiento.

Ahora bien, la consolidación de la relación entre salud mental y desarrollo positivo del adolescente deja entrever por lo menos tres aspectos que sirven para orientar el desarrollo de procesos de construcción de conocimiento en torno a la adolescencia en la actualidad. 
El primero, se refiere al reconocimiento del adolescente como sujeto sano mentalmente, con lo que se busca enfatizar en las condiciones necesarias para el desarrollo del bienestar y de sus capacidades en los diferentes ámbitos de la vida (familiar, educativa, laboral, comunitaria); esta perspectiva propone recurrir a modelos teóricos que definan la salud mental positiva.

El segundo, hace alusión al reconocimiento de los adolescentes como sujetos activos históricos y socialmente determinados, lo que implica validar su participación en la identificación de sus condiciones de vida, capacidades, potencialidades y satisfactores considerados necesarios para alcanzar un estado de salud mental positiva; imperativo necesario para trascender las posturas asistencialistas y adultocéntricas con las que se responde a sus necesidades y protege su salud mental.

El tercer aspecto, tiene que ver con la posibilidad de identificar cuáles son los ambientes y recursos que ofrecen satisfacción a las necesidades personales y sociales de los adolescentes, con la firme intención de garantizar el pleno goce de sus derechos y el desarrollo de capacidades de agenciar sus condiciones; otorgándoles un papel activo y protagonista en las transformaciones sociales y la construcción de tejido social.

La facultad de psicología de la Universidad CES y su grupo de investigación "Psicología, salud y sociedad" articulan dos de sus líneas de investigación: "Infancia, adolescencia y juventud" y "psicología y salud" en aras de responder a estas cuestiones; y ha logrado consolidar varios aprendizajes: la concepción del adolescente como sujeto de derechos, ubicado social e históricamente; el reconocimiento de la salud mental como un constructo que se define en la interdependencia entre bienestar, capacidades, funcionamientos y contribuciones sociales y se condiciona por los determinantes sociales; y la salud mental positiva como un campo de estudio que permite dar sentido a las apuestas institucionales por configurar enfoques integrados e integrales en salud mental.

Estos aprendizajes son coherentes con las directrices de la Organización Mundial de la Salud (2013) cuando plantea como objetivos de la estrategia de promoción de la salud mental, el empoderamiento de los individuos, el desarrollo de capacidades y el mejoramiento de la situación socioeconómica como base para garantizar la salud de las poblaciones, y además han permitido el encuentro de investigadores con otros sectores preocupados por la calidad de vida y el desarrollo humano de los adolescentes, constituyéndose en un horizonte y línea de trabajo posterior.

\section{Referencias}

Damon W. (2008). The Path to Purpose: Helping Our Children Find Their Calling in Life. New York: Simon and Schuster.

Frisch, M. B. (2000). Improving mental and physical health care through quality of life therapy and assessment. In E. Diener \& D. R. Rahtz (Eds.), Advances in quality of life: Theory and research (pp. 207-241). London: Kluwer

Herrera Santi, P. (1999). Principales factores de riesgo psicológicos y sociales en el adolescente. Revista Cubana de Pediatría, 71(1), 39-42. Recuperado de http:// scielo.sld.cu/scielo.php?script=sci arttext\&pid=S0034-75311999000100006\&l-

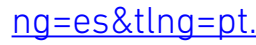

Hui, E.K, \& Tsang, S.K. (2012). Self-Determination as a Psychological and Positive Youth Development Construct. The Scientific World Journal, Article ID 759358. doi: https://doi.org/10.1100/2012/759358 
Kashdan, T.B, \& Mcknight, P.E. (2009). Origins of Purpose in Life: Refining our Understanding of a Life Well Lived. Psychol Top, 18(2), 303-16. Recuperado de https://westallen. typepad.com/files/origins-of-purpose-in-life.pdf

Larson, R. W. (2000). Toward a psychology of positive youth development. American Psychologist, 55(1), 170-183. https://doi.org/10.1037/0003-066X.55.1.170

Restrepo Ochoa, D. A., \& Jaramillo Estrada, J. C. (2012). Concepciones de salud mental en el campo de la salud pública. Rev. Fac. Nac. Salud Pública, 30(2), 202-211. Recuperado de http://www.scielo.org.co/pdf/rfnsp/v30n2/v30n2a09.pdf

Organización Mundial de la Salud -OMS-. (2013). Salud mental: un estado de bienestar. Recuperado de http://www.who.int/features/factfiles/mental health/es/

Organización Mundial de la Salud -OMS-. (s.f.). Salud de los adolescentes. Recuperado a partir de http://www.who.int/topics/adolescent health/es/

UNICEF. (2011). La salud mental del adolescente: Un desafío urgente para la investigación y la inversión. Recuperado de https://www.unicef.org/spanish/ sowc2011/pdfs/La-salud-mental-del-adolescente.pdf 